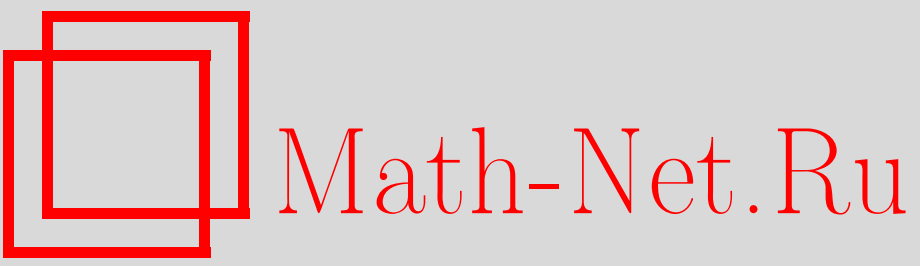

А. Кунду, Квантовые интегрируемые многоатомные модели, описывающие взаимодействие излучения с веществом с учетом и без учета приближения вращающейся волны, ТMФ, 2005, том 144, номер 1, 110-121

DOI: https://doi.org/10.4213/tmf1837

Использование Общероссийского математического портала Math-Net.Ru подразумевает, что вы прочитали и согласны с пользовательским соглашением

http://www.mathnet.ru/rus/agreement

Параметры загрузки:

IP: 54.196 .121 .252

26 апреля 2023 г., $18: 15: 35$ 


\section{КВАНТОВЫЕ ИНТЕГРИРУЕМЫЕ МНОГОАТОМНЫЕ МОДЕЛИ, ОПИСЫВАЮЩИЕ ВЗАИМОДЕЙСТВИЕ ИЗЛУЧЕНИЯ С ВЕЩЕСТВОМ С УЧЕТОМ И БЕЗ УЧЕТА ПРИБЛИЖЕНИЯ ВРАШАЮШЕЙСЯ ВОЛНЫ}

Построены и с помощью алгебраического бете-анзаца точно решены новые интегрируемые многоатомные модели, описывающие взаимодействие излучения с веществом с учетом и без учета приближения вращающейся волны (ПВВ). Модели, в которых учитывается ПВВ, строятся единообразным способом с помощью подхода, основанного на первичной модели (ancestor model). В случае рациональной $R$-матрицы получаются модели стандартного типа, описывающие взаимодействие излучения с веществом, в то время как случай тригонометрической $R$-матрицы соответствует их $q$-деформациям. Модели без учета ПВВ получаются для эллиптической $R$-матрицы в пределе модели Годена и большого спина.

Ключевые слова: интегрируемые многоатомные модели, приближение вращающейся волны, взаимодействие излучения с веществом.

\section{1. ВВЕДЕНИЕ}

Интересно отметить, что все реальные системы, такие как системы в квантовой оптике, индуцированные резонансным взаимодействием между атомом и квантованным лазерным полем, системы в резонаторной КЭД как в области СВЧ, так и в оптической области [1], [2], системы, состоящие из захваченного иона, взаимодействуюшего со своим движением центра масс и облучаемого лазерным пучком [3], [4], и т.п., содержашие сложные взаимодействия излучения с вешеством (ИВ), можно успешно описывать с помощью простых моделей, таких как модели Джейнса-Каммингса (ДжК) [5] и Бака-Сукумары (БС) [6]. Многие теоретические предсказания, сделанные на основании этих моделей, такие как вакуумное расшепление Раби, осцилляция Раби и ее квантовый коллапс и возрождение и т.д., были проверены в ходе экспериментов с мазерами и лазерами.

Квантованное поле излучения в таких простейших ИВ-моделях берется в виде одной бозонной моды: $h=b e^{-i \omega_{f} t}+b^{\dagger} e^{i \omega_{f} t}$, в то время как атом рассматривается как

${ }^{*}$ Saha Institute of Nuclear Physics, Theory Group, 1/AF Bidhan Nagar, Calcutta 700 064, India. E-mail: anjan@tnp.saha.ernet.in 
двухуровневая спиновая система с вектором поляризации $S=\sigma^{-} e^{-i \omega_{a} t}+\sigma^{+} e^{+i \omega_{a} t}$. Поэтому взаимодействие $(h \cdot S)$ содержит, вообще говоря, как быстро (с частотой $\omega_{f}+$ $\omega_{a}$ ), так и медленно (с частотой $\left|\omega_{f}-\omega_{a}\right|$ ) осциллирующие компоненты. Однако быстро осциллируюшей частью, как правило, пренебрегают, рассматривая аппроксимацию $\left|\omega_{f}-\omega_{a}\right| \ll \omega_{f}$, которая соответствует приближению врашаюшейся волны (ПВВ) и делает эту модель решаемой. Добавление членов, соответствующих свободному полю и атомному возбуждению, приводит к хорошо известной модели ДжК:

$$
H_{\text {ДжК }}=\omega_{f} b^{\dagger} b+\omega_{a} \sigma^{z}+\alpha\left(b^{\dagger} \sigma^{-}+b \sigma^{+}\right) .
$$

Однако этого приближения, которое применимо только вблизи точки резонанса $\omega_{f} \approx$ $\omega_{a}$, следует избегать в общем случае [7], когда должны появляться дополнительные члены, связанные с противоположно врашающейся волной (ПрВВ):

$$
H_{\Pi \mathrm{pBB}}=\beta\left(b^{\dagger} \sigma^{+}+b \sigma^{-}\right)
$$

Далее, для более точного описания физических ситуаций необходимо найти обобщения исходных моделей, т.е. рассмотреть их $q$-деформации [8], [9], ввести высшие нелинейности [4], [10], учесть многоатомность [2], [10], [11], рассмотреть межатомные взаимодействия и т.д. Однако если точные решения для моделей ДжК и БС, а также для их простых многоатомных расширений известны [12]-[14], то это, по-видимому, уже не так для большинства упомянутых выше обобшений. Точнее, интегрируемые модели как без учета ПВВ, так и с явным учетом межатомных взаимодействий не известны для большинства ИВ-моделей, если не считать нескольких недавних попыток таких построений в [15], [16]. Более того, хотя $q$-деформация, связанная с введением в систему анизотропии и специфической нелинейности, рассматривалась ранее для нескольких моделей [8], [9], их многоатомные и интегрируемые варианты неизвестны. Поэтому нахождение схемы построения интегрируемых ИВ-моделей, имеющих упомянутые выше желаемые свойства, действительно представляет собой сложную задачу.

\section{2. ОСНОВНЫЕ ЦЕЛИ И СТРАТЕГИЯ НАШЕГО ПОСТРОЕНИЯ}

Чтобы разрешить эту проблему, мы предлагаем в данной работе новые классы интегрируемых ИВ-моделей с учетом и без учетв ПВВ, которые являются точно решаемыми с помошью анзаца Бете. На основе собранных знаний [17] о решении квантового уравнения Янга-Бакстера (КУЯБ) в работе [18] была предложена единая конструкция интегрируемых моделей, связанная с лежащими в их основе алгебраическими структурами. Применяя подход, основанный на первичном операторе Лакса, мы предлагаем общую интегрируемую систему, различные редукции которой могут порождать ряд интегрируемых ИВ-моделей с явным учетом межатомных взаимодействий. Этот подход охватывает новые многоатомные обобшения моделей ДжК, БС и модели захваченного иона (ЗИ), а также их интегрируемые $q$-деформации с использованием квантовой групшы. Более того, поскольку построение наших моделей основано на алгебре Янга-Бакстера (ЯБ) обшего вида, мы можем также решить их единообразным способом с помощью бете-анзаца. Результаты, касаюшиеся наших моделей, учитываюших ПВВ, были недавно 
опубликованы в работе [19], и мы намереваемся дать здесь краткий обзор, а затем представить новые результаты, полученные для моделей без учета ПВВ.

Наша стратегия при построении моделей с учетом ПВВ заключается в том, чтобы начать с комбинации бозонного оператора Лакса $L^{s}(\lambda)$, описьвающего излучение или осциллирующую моду, и $N_{a}$ спиновых операторов Лакса $L_{j}^{S}(\lambda)$, соответствующих системе многих атомов. По построению глобальный оператор монодромии должен удовлетворять КУЯБ

$$
R(\lambda-\mu) T(\lambda) \otimes T(\mu)=(I \otimes T(\mu))(T(\lambda) \otimes I) R(\lambda-\mu)
$$

что приводит к условию интегрируемости $[\tau(\lambda), \tau(\mu)]=0$ и к набору взаимно коммутируюших сохраняюшихся операторов $\left[C_{a}, C_{b}\right]=0$, получаемых из разложения $\tau(\lambda)=\operatorname{tr} T(\lambda)=\sum_{a} C_{a} \lambda^{a}[20] . \quad$ Для построения бозонного оператора Лакса мы начнем с оператора Лакса обшего вида [18], связанного с квадратичной алгеброй или ее квантовой деформацией и порождающего через различные бозонные реализации $L^{s}(\lambda)$ различные интегрируемые системы. ИВ-модели стандартного типа, которые мы строим, связаны с алгебрами типа алгебр Ли, однако квадратичными, а также с рациональной $R$-матрицей спиновой $X X X$-цепочки [20], в то время как их $q$-деформации связаны с квантовой квадратичной алгеброй и тригонометрической $R$-матрицей $X X Z$-цепочки [21].

Однако при построении интегрируемых ИВ-моделей без учета ПВВ описанный выше подход не работает из-за трудности бозонизации алгебры Склянина. Поэтому для таких моделей, которые связаны с эллиптической $R$-матрицей спиновой $X X Z$-цепочки, нам придется изменить стратегию и перейти к пределу модели Годена, а также к пределу больших спинов.

\section{3. ИНТЕГРИРУЕМЫЕ ИВ-МОДЕЛИ С УЧЕТОМ ПВВ}

Вначале сосредоточимся на стандартных ИВ-моделях и напомним, что в рациональном случае первичный $(2 \times 2)$-оператор Лакса имеет вид [18]

$$
L^{s}(\lambda)=\left(\begin{array}{cc}
c_{1}^{0}\left(\lambda+s^{3}\right)+c_{1}^{1} & s^{-} \\
s^{+} & c_{2}^{0}\left(\lambda-s^{3}\right)-c_{2}^{1}
\end{array}\right)
$$

при этом операторы s удовлетворяют квадратичной алгебре

$$
\left[s^{+}, s^{-}\right]=2 m^{+} s^{3}+m^{-}, \quad\left[s^{3}, s^{ \pm}\right]= \pm s^{ \pm}, \quad\left[m^{ \pm}, \cdot\right]=0
$$

где центральные элементы $m^{ \pm}$выражаются через элементы, входяшие в $(3): m^{+}=$ $c_{1}^{0} c_{2}^{0}, m^{-}=c_{1}^{1} c_{2}^{0}+c_{1}^{0} c_{2}^{1}$. Заметим, что алгебра (4) определяется самим КУЯБ, решение которого определяет рациональную $R$-матрицу. Элементы $m^{ \pm}, c_{i}^{a}$ могут иметь тривиальные собственные значения и в общем случае не могут быть устранены простым преобразованием алгебры к $s l(2)$. На самом деле определенные способы выбора этих 
элементов в случае обшего положения ограничивают уравнение (4) до различных известных алгебр, таких как его подалгебры, без возникновения какого бы то ни было сингулярного предела. Например, можно получить

$$
\begin{aligned}
& \mathbf{s}=\mathbf{S} \in \operatorname{su}(2) \text { при } m^{+}=1, \quad m^{-}=0, \\
& \mathbf{s} \in \operatorname{su}(1,1) \text { при } m^{+}=-1, \quad m^{-}=0, \\
& \mathbf{s} \rightarrow\left(b, b^{\dagger}\right) \text { (бозоны) при } m^{+}=0, m^{-}=-1, \\
& \mathbf{s} \rightarrow(x, p) \quad \text { (канонические переменные) при } m^{+}=m^{-}=0,
\end{aligned}
$$

и в соответствуюших случаях из (3) получаются соответствуюшие операторы Лакса. В случае (5а), выбирая $c_{a}^{0}=1$ и $c_{1}^{1}=-c_{2}^{1}=c_{j}$, мы находим из (3) хорошо известньй спиновый оператор Лакса $L_{j}^{S}(\lambda), j \in\left[1, N_{a}\right]$, описываюший $N_{a}$ атомов с параметрами неоднородности $c_{j}$, в то время как другие случаи могут приводить к различным типам бозонных операторов Лакса, связанных с излучательной модой.

Комбинируя спиновый и бозонные операторы Лакса:

$$
T(\lambda)=L^{s}(\lambda) \prod_{j}^{N_{a}} L_{j}^{S}(\lambda),
$$

мы можем теперь с помошью описанной выше стандартной процедуры построить набор коммутируюших сохраняюшихся операторов. Самый простой из них имеет вид

$$
C_{N_{a}}=s^{3}+\sum_{j}^{N_{a}} S_{j}^{z},
$$

а старшие учитывают все более старшие многочастичные взаимодействия. Следующий сохраняюшийся оператор в наборе $\alpha C_{N_{a}-1}$, где $\alpha$ - произвольная константа взаимодействия, можно определить как гамильтониан нашей обобшенной ИВ-системы:

$$
\begin{aligned}
H_{\text {ИВ }} & =H_{d}+H_{S s}+H_{S S}, \\
H_{d} & =\omega_{f} s^{3}+\sum_{j}^{N_{a}} \omega_{a j} S_{j}^{z}, \\
H_{S s} & =\alpha \sum_{j}^{N_{a}}\left(s^{+} S_{j}^{-}+s^{-} S_{j}^{+}+\left(c_{1}^{0}+c_{2}^{0}\right) s^{3} S_{j}^{z}\right), \\
H_{S S} & =\alpha \sum_{i<j}\left(\left(c_{1}^{0}+c_{2}^{0}\right) S_{i}^{z} S_{j}^{z}+c_{1}^{0} S_{i}^{-} S_{j}^{+}+c_{2}^{0} S_{i}^{+} S_{j}^{-}\right) .
\end{aligned}
$$

Здесь $H_{S s}$ описьвает ИВ-взаимодействие, а $H_{S S}$ - взаимодействие вещества с вешеством; $\mathbf{S}_{j}, j=1,2, \ldots, N_{a}$, соответствуют набору $N_{a}$ атомов, каждый из которых имеет $2 s+1$ уровней, и удовлетворяют $s u(2)$-алгебре. С другой стороны, $\mathbf{s}$ обозначает излучательную или колебательную моду и удовлетворяет более обшей алгебре (4). В уравнениях (6) частота излучения $\omega_{f}$ и атомные частоты $\omega_{a j}, j=1,2, \ldots, N_{a}$, определяются параметрами неоднородности оператора Лакса как

$$
\omega_{f}=\sum_{j} w_{j}, \quad w_{j}=\alpha\left(c_{1}^{0}-c_{2}^{0}\right) c_{j}, \quad \omega_{a j}=\omega_{f}-w_{j}+\alpha\left(c_{1}^{1}+c_{2}^{1}\right) .
$$


Заметим, что эрмитовость этого интегрируемого гамильтониана существенно зависит от выбора входящих в него произвольных параметров.

Замечательно, что общая модель (6) единообразным способом приводит к новой серии интегрируемых многоатомных моделей БС, ДжК и ЗИ в частных случаях (5б), (5в) и (5г). Например, в случае (5б), выбирая $c_{1}^{0}=-c_{2}^{0}=1, c_{1}^{1}=c_{2}^{1} \equiv c$, получаем уравнение

$$
H_{b s}=\omega_{f} s^{3}+\sum_{j}^{N_{a}}\left(\omega_{a j} S_{j}^{z}+\alpha\left(s^{+} S_{j}^{-}+s^{-} S_{j}^{+}\right)\right)+\alpha \sum_{i<j}^{N_{a}}\left(S_{i}^{-} S_{j}^{+}-S_{i}^{+} S_{j}^{-}\right),
$$

которое в случае бозонной реализации $s u(1,1)$ вида $s^{+}=\sqrt{N} b^{\dagger}, s^{-}=b \sqrt{N}, s^{3}=$ $N+1 / 2$ и со спиновым оператором $s$, выбранным в виде $\vec{S}=(1 / 2) \sum_{k}^{2 s} \vec{\sigma}_{k}$, представляет собой новую интегрируемую многоатомную ИВ-модель с межатомными взаимодействиями и невырожденными атомными частотами. Заметим, что, как показывают наши вычисления для этой интегрируемой модели [19], хотя явный вид той части гамильтониана, которая отвечает за межатомные взаимодействия, оказывается здесь неэрмитовым, собственные значения энергии могут быть вешественными или комплексно-сопряженными в зависимости от интервала входящих в уравнение частотных параметров. При $N_{a}=1$, когда взаимодействие вешества с вешеством исчезает, естественным образом воспроизводится известная модель [14].

Аналогично можно рассмотреть случай (5в), выбрав $c_{1}^{0}=\alpha, c_{2}^{0}=0, c_{1}^{1} \equiv c, c_{2}^{1}=$ $-\alpha^{-1}$. Это дает возможность непосредственно получить из алгебры бозонную реализацию $s^{-}=b, s^{+}=b^{\dagger}, s^{3}=b^{\dagger} b$, а из тех же уравнений (3) и (6) - интегрируемую многоатомную модель ДжК, учитываюшую взаимодействие вешества с вешеством. Явный вид гамильтониана легко можно вывести из обшей модели (6), подставляя в нее приведенные значения параметров и бозонные операторы.

Теперь можно рассмотреть случай (5г), фиксируя значения параметров в виде $c_{1}^{0}=$ $-1, c_{1}^{1} \equiv c, c_{2}^{0}=c_{2}^{1}=0$, и построить самосогласованную реализацию с использованием канонических переменных вида $s^{ \pm}=e^{\mp i x}, s^{3}=p+x$. В результате из уравнений (6) получается новая интегрируемая многоатомная модель ЗИ

$$
H_{\text {зи }}=\left(\omega_{a}-\omega_{f}\right) S^{z}+S^{z 2}+\alpha\left(e^{-i x} S^{+}+e^{i x} S^{-}\right)+H_{x p},
$$

где $H_{x p}=(1 / 2)\left(p^{2}+x^{2}\right)+x p, \quad \vec{S}=(1 / 2) \sum_{k} \vec{\sigma}_{k}$. Здесь мы привели только случай $N_{a}=1$, который уже демонстрирует наличие полной экспоненциальной нелинейности без обычной линейной аппроксимации. Однако хотя такие модели и являются интегрируемыми, их трудно решать с помошью стандартного анзаца Бете из-за отсутствия псевдовакуума (как в цепочке Тоды).

При построении интегрируемых q-деформированных ИВ-моделей используется такая же стратегия; только здесь следует начинать с тригонометрической $R$-матрицы и связанного с ней оператора Лакса первичной модели, содержащего обобщенные $q$-деформированные операторы, удовлетворяющие алгебре [18]

$$
\left[s_{q}^{3}, s_{q}^{ \pm}\right]= \pm s_{q}^{ \pm}, \quad\left[s_{q}^{+}, s_{q}^{-}\right]=\frac{1}{2 \sin \alpha}\left(M^{+} \sin \left(2 \alpha s_{q}^{3}\right)-i M^{-} \cos \left(2 \alpha s_{q}^{3}\right)\right)
$$


где $q=e^{i \alpha}$ и $M^{ \pm}$- центральные элементы. Поэтому с помошью таких же построений можно вывести нашу обшую $q$-деформированную интегрируемую систему с гамильтонианом

$$
\begin{aligned}
H_{q \text { Ив }} & =H_{d}+\left(s_{q}^{+} S_{q}^{-}+s_{q}^{-} S_{q}^{+}\right) \sin \alpha, \\
H_{d} & =-i c_{0} \cos (\alpha X)+c \sin (\alpha X), \quad X=\left(s_{q}^{3}-S_{q}^{3}+\omega\right),
\end{aligned}
$$

которая представляет собой новый класс ИВ-моделей, в которых $\mathbf{S}_{q}$ принадлежат квантовой группе $s u_{q}(2)$, a $\mathbf{s}_{q}$ принадлежат более обшей квантовой алгебре (10). Здесь мы для простоты пренебрегли явными межатомными взаимодействиями.

Заметим, что квадратичная алгебра (10) также следует из КУЯБ для случая тригонометрической $R$-матрицы, и аналогично рациональному случаю можно получить различные “подалгебры” алгебры (10) как $q$-деформации модели (5):

$$
\begin{aligned}
& \mathbf{s}_{q}=\mathbf{S}_{q} \in s u_{q}(2), \\
& \mathbf{s}_{q} \in s u_{q}(1,1), \\
& \mathbf{s}_{q} \rightarrow\left(b_{q}, b_{q}^{\dagger}\right) \quad(q \text {-бозоны }), \\
& \mathbf{s}_{q} \rightarrow(x, p) \quad \text { (канонические переменные). }
\end{aligned}
$$

Аналогичным образом мы можем порождать интегрируемые $q$-деформации построеных выше моделей. Например, случай $\left(5^{\prime}\right.$ б) при $\mathbf{s}_{q} \in s u_{q}(1,1)$ допускает реализацию с помошью $q$-осциллятора $[9]: s_{q}^{+}=\sqrt{[N]_{q}} b_{q}^{\dagger}, s_{q}^{-}=b_{q} \sqrt{[N]_{q}}, s_{q}^{3}=N+1 / 2$. Используя эти результаты, а также оператор квантового спина $\mathbf{S}_{q}$, представленный на $2 s=N_{a}$ атомах с помощью своего копроизведения [21],

$$
S_{q}^{ \pm}=\sum_{j}^{2 s} q^{-\sum_{k<j} \sigma_{k}^{z}} \sigma_{j}^{ \pm} q^{\sum_{l>j} \sigma_{l}^{z}}, \quad S^{z}=\sum_{j}^{2 s} \sigma_{j}^{z},
$$

можно получить из (11) интегрируемую q-деформированную многоатомную модель БC. При $s=1 / 2$, т.е. в случае одиночного атома, получаем интегрируемую версию описанной ранее модели [9].

Аналогичным образом в случае $\left(5^{\prime}\right.$ в), положив $s_{q}^{+}=b_{q}^{\dagger}, s_{q}^{-}=b_{q}, s_{q}^{3}=N$, можно построить новую интегрируемую q-деформащию модели ДжК, а в случае $\left(5^{\prime}\right.$ г) реализация через канонические операторы, как и ранее, дает в результате интегрируемую q-деформированнул модель ЗИ.

Мы снова подчеркиваем, что все предложенные нами интегрируемые ИВ-модели, аналогичные по своему единообразному построению, допускают точные решения методом бете-анзаца также единообразным путем. Более подробную информацию об описанных выше моделях и их точных решениях можно найти в работе [19].

\section{4. ИНТЕГРИРУЕМЫЕ ИВ-МОДЕЛИ БЕЗ УЧЕТА ПВВ}

Основная идея, используемая при построении интегрируемых моделей, описываюших ИВ-взаимодействие с учетом ПВВ, заключается, как мы объясняли выше, в том, чтобы сначала представить часть, соответствующую веществу, спиновой $X X X$ - или $X X Z$-цепочкой с произвольными спинами, а затем для построения части, относяшейся 
к излучению, заменить один из спиновых операторов на различные разрешенные бозонные реализации. Первый случай относится к рациональному классу, когда спины удовлетворяют алгебре типа алгебры Ли, тогда как второй случай сводится к тригонометрическому, когда операторы высших спинов удовлетворяют квантовой алгебре. $\mathrm{K}$ счастью, обе эти алгебры допускают бозонные реализации, позволяющие получить требуемую излучательную моду.

Кажется, что при построении более общих интегрируемых моделей с дополнительными ПрВВ-членами (такими как (2) и его многоатомные обобшения) можно было бы применить ту же самую идею, но только для спиновой $X Y Z$-цепочки. Однако в этом анизотропном случае, соответствуюшем эллиптической $R$-матрице, операторы высших спинов удовлетворяют алгебре Склянина [22], для которой, к сожалению, бозонная реализация неизвестна. В поисках выхода из такой ситуации можно заметить, что алгебра Склянина удачным образом снова редуцируется к обычной $s u(2)$-алгебре в эллиптическом пределе Годена [23], допускающем небходимую бозонную реализацию. Однако прямая бозонная реализация одного спинового оператора в моделях Годена снова невозможна, поскольку в результате получаются или неинтегрируемые, или нефизические модели. Ситуацию в конце концов спасает дальнейший переход к пределу $s \rightarrow \infty$ для бозонной моды, который приводит к желаемой интегрируемой многоатомной модели типа модели ДжК с нетривиальными ПрВВ-членами [24]. Следует заметить, что аналогичньй предел больших спинов был недавно использован при построении интегрируемой многоатомной ДжК-модели, но учитываюшей ПВВ [16].

4.1. Эллиптическая модель Годена. Поскольку первым шагом в нашем построении является эллиптическая модель Годена (ЭМГ), мы дадим краткий обзор связанных с ней результатов, полученных в работе [23]. ЭМГ возникает из неоднородной спиновой $X Y Z$-модели в пределе $\alpha \rightarrow 0$, где $q=e^{i \alpha}$, и поэтому все соответствующие объекты, такие как оператор Лакса, $R$-матрица, сохраняющиеся величины, а также важные формулы, используемые в методе бете-анзаца, как то: собственные функции, собственные значения и уравнения Бете, и т.д. можно непосредственно вывести из соответствующих величин $X Y Z$-модели [25] при переходе к тому же пределу. Оператор Лакса с параметрами неоднородности $z_{n}$ и $r$-матрицу в ЭМГ можно выразить через эллиптические функции таким образом [23]:

$$
L_{n}(\lambda)=\sum_{a=1}^{3} w_{a}\left(\lambda-z_{n}\right) S_{n}^{a} \otimes \sigma^{a}, \quad r(\lambda)=\sum_{a=1}^{3} w_{a}(\lambda) \sigma^{a} \otimes \sigma^{a}, \quad n=0,1, \ldots, N_{a},
$$

где

$$
w_{1}(\lambda)=\frac{\operatorname{cn}(\lambda)}{\operatorname{sn}(\lambda)}, \quad w_{2}(\lambda)=\frac{\operatorname{dn}(\lambda)}{\operatorname{sn}(\lambda)}, \quad w_{3}(\lambda)=\frac{1}{\operatorname{sn}(\lambda)} .
$$

Операторы (12) удовлетворяют квазиклассическому УЯБ:

$$
\left[L_{n}(\lambda) \otimes L_{n}(\mu)\right]=\left[r(\lambda-\mu),\left(L_{n}(\lambda) \otimes I+I \otimes L_{n}(\mu)\right)\right]
$$

полученному из КУЯБ в пределе $\alpha \rightarrow 0$. Взаимно коммутирующие сохраняюшиеся величины $H_{n}$ в ЭМГ находятся путем разложения коэффициентов $X Y Z$-трансфер-матрицы

$$
\tau_{x y z}(\lambda, \alpha)=I+\alpha^{2} \tau^{(2)}(\lambda)+O\left(\alpha^{3}\right)
$$


следуюшим образом:

$$
\tau^{(2)}\left(\lambda \rightarrow z_{n}\right)=H_{n}=\sum_{a, m \neq n} w_{a}\left(z_{n}-z_{m}\right) S_{n}^{a} S_{m}^{a},
$$

где $\sum_{n} H_{n}=0$.

Для наших целей важно заметить, что операторы высших спинов $S_{n}^{a}$, появляюшиеся в модели Годена (12), редуцированы из генераторов алгебры Склянина просто к операторам

$$
\left[S_{n}^{+}, S_{m}^{-}\right]=2 \rho \delta_{n m} S_{n}^{3}, \quad\left[S_{n}^{3}, S_{m}^{ \pm}\right]= \pm \delta_{n m} S_{n}^{ \pm},
$$

где $\rho= \pm$ соответствует $s u(2)$ (или $s u(1,1)$ ). Напомним, что при построении стандартных ИВ-моделей с учетом ПВВ мы использовали спиновые операторы для $N_{a}$ атомов в качестве генераторов алгебры $s u(2)$, тогда как для поля излучения мы рассматривали бозонные реализации различных алгебраических структур в (5). Для ЭМГ (12), однако, кроме $s u(2)$, единственной другой возможной алгеброй в соответствии с (15) является алгебра $s u(1,1)$. И даже этот факт мы не можем использовать, поскольку различные алгебры не могут быть смешаны в интегрируемой модели Годена. С другой стороны, если мы выберем алгебру $s u(2)$ для всех операторов и воспользуемся преобразованием Холстейна-Примакова (ПХП) $S_{0}^{+}=b^{\dagger} \sqrt{s_{0}-N}, S_{0}^{-}=\sqrt{s_{0}-N} b, S_{0}^{3}=N-s_{0} / 2$, $N=b^{\dagger} b$ для бозонизации моды излучения, то хотя мы и сохраним интегрируемость, но при $N>s_{0} / 2$ получим нефизический гамильтониан, а $S_{0}^{ \pm}$станет неэрмитовым!

4.2. Предел большого спина. К счастью, описанные вьше трудности можно снова преодолеть, если далее рассматривать предел большого спина $s_{0} \rightarrow \infty$ для поля излучения, который редуцирует ПХП к виду

$$
S_{0}^{+}=\frac{1}{\epsilon} b^{\dagger}, \quad S_{0}^{-}=\frac{1}{\epsilon} b, \quad S_{0}^{3}=-\frac{1}{2 \epsilon^{2}}, \quad \epsilon=\frac{1}{\sqrt{s_{0}}} \rightarrow 0,
$$

где сохранены члены до порядка $O(1 / \epsilon)$.

Теперь для вывода интегрируемых моделей, описывающих ИВ-взаимодействие без учета ПВВ, мы заменим спиновые операторы при $n=0$ бозонами для излучательной моды в уравнении (14), используя соотношения (16), и выберем произвольные параметры при $n=0, j$ в виде

$$
z_{0}=K+\epsilon x_{0}, \quad z_{j}=\epsilon x_{j}, \quad j=1, \ldots, N_{a},
$$

где $K$ - эллиптический интеграл [25], связанный с периодом эллиптических функций. Такой скейлинг параметра необходим для вывода набора сохраняюшихся взаимно коммутируюших операторов. Мы также используем симметрию алгебры $s u(2)$, чтобы для удобства следуюшим образом переопределить постоянные взаимодействия в уравнении (14): $w_{1}\left(z_{j}-z_{k}\right) \leftrightarrow w_{3}\left(z_{j}-z_{k}\right), w_{2}\left(z_{j}-z_{k}\right)=-w_{2}\left(z_{j}-z_{k}\right)$ и аналогично для $w_{a}\left(z_{0}-\right.$ $\left.z_{k}\right)$. Таким образом, в пределе $\epsilon \rightarrow 0$ с использованием таких свойств, как $w_{ \pm}\left(z_{0}-\right.$ 
$\left.z_{k}\right)=-w_{ \pm}\left(z_{k}-z_{0}\right)$, и свойств эллиптических функций [25] переопределенные константы взаимодействия в требуемом порядке редуцируются к виду

$$
w_{1}(0 j) \rightarrow 1, \quad w_{2}(0 j) \rightarrow k^{\prime}, \quad w_{3}(0 j) \rightarrow 0, \quad w_{a}(j k) \rightarrow \frac{1}{\epsilon\left(x_{j}-x_{k}\right)}, \quad a=1,2,3,
$$

с эллиптическим модулем $k^{\prime}=\sqrt{1-k^{2}}$. Это окончательно редуцирует эллиптический гамильтониан Годена в главном порядке к интегрируемой модели, описываюшей ИВ-взаимодействие, с взаимно коммутирующими гамильтонианами $\left[H_{j}, H_{k}\right]=0$, где

$$
H_{j}=\omega_{a} S_{j}^{3}+H_{j}^{(b S)}+H_{j}^{(S S)}, \quad j=1, \ldots, N_{a} .
$$

Здесь

$$
H_{j}^{(b S)}=\Omega_{+}\left(b S_{j}^{+}+b^{\dagger} S_{j}^{-}\right)+\Omega_{-}\left(b^{\dagger} S_{j}^{+}+b S_{j}^{-}\right), \quad \Omega_{ \pm}=1 \pm k^{\prime},
$$

описывает ИВ-взаимодействие с явным учетом ПрВВ-членов, а

$$
H_{j}^{(S S)}=\sum_{k \neq j}^{N_{a}} \frac{1}{x_{j}-x_{k}}\left(S_{j}^{+} S_{k}^{-}+S_{j}^{-} S_{k}^{+}+S_{j}^{3} S_{k}^{3}\right)
$$

отвечает за межатомные взаимодействия с неоднородными постоянными взаимодействия, которые выражаются через $x_{j}$. Эти произвольные параметры можно таким образом согласовать, чтобы напряженности, возникающие при взаимодействиях, уменьшались при возрастании расстояния между атомами. Заметим, что соотношение (21) является эрмитовым и интересным образом не содержит взаимодействий $X Y Z$-типа, которые позволяют добавлять к гамильтониану (19) член с атомной инверсией с произвольной частотой $\omega_{a}$. Нетрудно видеть, что константа взаимодействия $\Omega_{-}$обрашается в нуль при $k=0$, воспроизводя тем самым предыдущий результат, полученный в ПВВ [16].

Различные комбинации гамильтонианов (19) с произвольными константами взаимодействия могут приводить к различным интегрируемым многоатомным моделям, описъвающим ИВ-взаимодействие, без учета ПВВ. Например, если просто взять

$$
H_{0}=\sum_{j} H_{j}=\sum_{j}^{N_{a}}\left(\omega_{a} S_{j}^{3}+\Omega_{+}\left(b S_{j}^{+}+b^{\dagger} S_{j}^{-}\right)+\Omega_{-}\left(b^{\dagger} S_{j}^{+}+b S_{j}^{-}\right)\right),
$$

то, поскольку $\sum_{j} H_{j}^{(S S)}=0$, мыпридем к интегрируемой модели типа модели Тависа-Каммингса с нетривиальными ПрВВ-членами. Такие модели обычно считаются нерешаемыми без дальнейших аппроксимаций [7]. Однако ниже мы покажем, что они являются точно решаемыми с помощью бете-анзаца. Для того чтобы сделать модель более физической, мы должны добавить полевой член $\omega_{f} b^{\dagger} b$ в гамильтониан (22). Полагая частоту поля $\omega_{f}$ малой по сравнению с $\omega_{a}$, можно рассматривать этот дополнительный член как пертурбативный относительно исходной решаемой интегрируемой бете-модели. Интересно заметить, что особенно в этом пределе малой частоты поля ПВВ становится плохой аппроксимацией и ПрВВ-члены, которые мы рассматривали, оказываются более важными. Более подробное описание построения наших ИВ-моделей без учета ПВВ представлено в работе [24]. 


\section{5. ТОЧНЫЕ РЕШЕНИЯ МЕТОДОМ БЕТЕ-АНЗАЦА}

В основном методе алгебраического бете-анзаца (АБА) след матрицы монодромии $\tau(\lambda)=\operatorname{tr} T(\lambda)$ порождает сохраняюшиеся операторы, тогда как внедиагональные элементы $T_{21}(\lambda) \equiv B(\lambda)$ и $T_{12}(\lambda) \equiv C(\lambda)$ действуют как операторы рождения и уничтожения псевдочастиц с $M$-частичным состоянием, задаваемым как $|M\rangle_{B}=$ $B\left(\lambda_{1}\right) \ldots B\left(\lambda_{M}\right)|0\rangle$, и с псевдовакуумом $|0\rangle$, определяемым как $C(\lambda)|0\rangle=0$. Основная цель АБА [20] состоит в том, чтобы найти решение для собственного значения: $\tau(\lambda)|M\rangle_{B}=\Lambda\left(\lambda,\left\{\lambda_{a}\right\}\right)|M\rangle_{B}$.

Все наши модели, учитываюшие ПВВ, построенные единообразным способом с помощью схемы, соответствуюшей первичной модели, могут быть также единообразно решены на основе метода АБА в его стандартной формулировке [20]. Опуская детали [19], сосредоточимся на АБА-решении наших интегрируемых моделей без учета ПВВ (19)-(22). При выводе АБА-соотношений для этих моделей следует повторить шаги, сделанные при получении выражений для их гамильтонианов. То есть мы должны начать с АБАсоотношений для спиновой $X Y Z$-цепочки произвольных спинов, где для определения модифицированного псевдовакуума [25], [26] следует использовать калибровочно преобразованные операторы и состояния. Если тщательно рассмотреть предел $\alpha \rightarrow 0$, то можно получить соответствующие АБА-соотношения для ЭМГ [23]. Если в этих соотношениях взять предел больших спинов для излучательной моды, то мы в конще концов найдем собственные значения и уравнения Бете для наших интегрируемых моделей без учета ПВВ.

Начнем поэтому с ЭМГ и, используя ключевые АБА-соотношения из [23], получим энергетический спектр в виде

$$
E_{n}^{\ni \mathrm{M \Gamma}}=2 s_{n} \theta_{11}^{\prime}(0)\left(i \pi \nu+\sum_{b=1}^{M} \frac{\theta_{11}^{\prime}\left(z_{n}-\lambda_{b}\right)}{\theta_{11}\left(z_{n}-\lambda_{b}\right)}+\sum_{m \neq n}^{M} s_{m} \frac{\theta_{11}^{\prime}\left(z_{n}-z_{m}\right)}{\theta_{11}\left(z_{n}-z_{m}\right)}\right)
$$

с произвольными значениями спина $s_{n}$ операторов в $n$-м узле и с эллиптической функцией $\theta_{11}$; здесь число возбуждений $M=\sum_{n} s_{n}$ больше не является произвольным. Можно найти соответствуюшие уравнения Бете [23] в виде

$$
\sum_{n=1}^{N} s_{n} \frac{\theta_{11}^{\prime}\left(\lambda_{a}-z_{n}\right)}{\theta_{11}\left(\lambda_{a}-z_{n}\right)}=-i \pi \nu+\sum_{b \neq a}^{M} \frac{\theta_{11}^{\prime}\left(\lambda_{a}-\lambda_{b}\right)}{\theta_{11}\left(\lambda_{a}-\lambda_{b}\right)} .
$$

Выбирая параметры неоднородности при $n=0, j$ как в уравнении (17), вводя два ряда параметров Бете со скейлингами $\lambda_{a}=\epsilon l_{a}, a=1, \ldots, N_{a} / 2$, и $\lambda_{\alpha}^{0}=\epsilon l_{\alpha}^{0}+K, \alpha=$ $1, \ldots, s_{0}$, и используя некоторые свойства $\theta_{11}$-функции, из соотношения (23) при $n=j$ можно теперь получить точное собственное значение для нашей модели, описываюшей ИВ-взаимодействие, без учета ПВВ. Таким образом, в пределе больших спинов (или, что эквивалентно, при $\epsilon \rightarrow 0$ ) для поля излучения с гамильтонианом (19) мы находим значение энергии

$$
E_{j}^{\Pi p B B}=\theta_{11}^{\prime}(0)\left(\omega_{a}+\sum_{b=1}^{N_{a} / 2} \frac{1}{\left(x_{j}-l_{b}\right)}+\frac{1}{2} \sum_{k \neq j}^{N_{a}} \frac{1}{\left(x_{j}-x_{k}\right)}+\frac{\theta_{10}^{\prime \prime}}{\theta_{10}}(0)\left(2 x_{j}-x_{0}-l^{0}\right)\right),
$$


где, чтобы получить определенное решение $l^{0}=x_{0}$, мы предположили, что параметр вырождения имеет значения $l_{\alpha}^{0}=l^{0}$ для всех $\alpha=1, \ldots, s_{0}$. Чтобы найти оставшиеся параметры Бете $\left\{l_{a}\right\}$, необходимо решить следуюшее уравнение Бете, полученное редукцией уравнения (24):

$$
\frac{1}{2} \sum_{k=1}^{N_{a}} \frac{1}{\left(x_{k}-l_{a}\right)}=\omega_{a}+\sum_{b \neq a}^{N_{a} / 2} \frac{1}{\left(l_{b}-l_{a}\right)}, \quad a=1, \ldots, \frac{N_{a}}{2}
$$

для заданного набора произвольных невырожденных параметров неоднородности $x_{j}$, $j=1, \ldots, N_{a}$. Заметим, что от значений $l^{0}=x_{0}$ в уравнении $(25)$ можно избавиться с помощью сдвига параметров $x_{j}$ и $l_{a}$.

Взяв сумму в уравнении (25) и используя для упрошения уравнение Бете (26), можно также легко получить точные собственные значения для нашей модели Тависа-Каммингса (22) с явными ПрВВ-членами.

\section{6. ЗАКЛЮЧИТЕЛЬНЫЕ ЗАМЕЧАНИЯ}

В реальных моделях, описываюших ИВ-взаимодействие, при наличии многих атомов должны появляться межатомные взаимодействия. Более того, в общем случае необходимо избегать приближения ПрВВ, по крайней мере вне точки резонанса. Однако если не нарушать свойство интегрируемости, эти важные случаи нельзя включать в рассмотрение в большинстве предложенных ранее решаемых моделей. Нами предложены новые интегрируемые модели, где оба этих случая можно объединить, сохраняя при этом интегрируемость системы, и получить точные решения с помощью бете-анзаца. Для моделей, учитываюших ПВВ, найден ряд интегрируемых многоатомных моделей с учетом межатомных взаимодействий, откуда получаются обобшения моделей ДжК, БС и модели ЗИ, а также их $q$-деформации. Эти модели относятся к рациональному или тригонометрическому классу, и их можно точно решить единообразным способом.

Нами также впервые построены точно решаемые многоатомные модели, описываюшие ИВ-взаимодействие, без учета ПВВ, близкие к физическим системам, содержашим требуемые члены, соответствуюшие случаю ПрВВ. Мы получили этот результат, взяв предел больших спинов для поля излучения в ЭМГ. Однако в отличие от моделей с учетом ПВВ, где нам удалось построить модели различного типа, описывающие ИВ-взаимодействие, наши успехи при построении моделей без учета ПВВ ограниченны, здесь мы смогли получить только модели типа модели ДжК с ПрВВ-членами. Более того, в таких интегрируемых моделях члены, соответствующие полю возбуждения, не появляются естественным путем и должны быть включены пертурбативным образом в точный результат, полученньй с помощью бете-анзаца.

Отождествление моделей с реальными системами и экспериментальная проверка представленных в настоящей работе результатов, особенно в экспериментах с многоатомными микролазерами [27] или с ионами, захваченными вне точки резонанса [7], могли бы оказаться важными применениями. 


\section{Список литературы}

[1] G. Rempe, H. Walther, N. Klein. Phys. Rev. Lett. 1987. V. 58. P. 353; G. Rempe, F. Schmidt-kaler, H. Walther. Phys. Rev. Lett. 1990. V. 64. P. 2783.

[2] M. Raizen R. J. Thompson, R. J. Brecha et al. Phys. Rev. Lett. 1989. V. 63. P. 240.

[3] C. A. Blockley, D. F. Walls, H. Risken. Europhys. Lett. 1992. V. 17. P. 509.

[4] W. Vogel, R. de Mitos Filho. Phys. Rev. A. 1995. V. 52. P. 4214.

[5] E. T. Jaynes, F. W. Cummings. Proc. IEEE. 1963. V. 51. P. 89.

[6] B. Buck, C. V. Sukumar. Phys. Lett. A. 1981. V. 81. P. 132.

[7] R. Angelo, M. K. Furuya, M. C. Nemes, G. Q. Pellegrino. Phys. Rev. A. 2001. V. 64. P. 043801.

[8] V. Buzek. J. Mod. Opt. 1992. V. 39. P. 949.

[9] M. Chaichian, D. Ellinas, P. Kulish. Phys. Rev. Lett. 1990. V. 65. P. 980.

[10] H.-S. Zheng, L. M. Kuang, K. L. Gao. Jaynes-Cummings model dynamics in two trapped ions. quant-ph/0106020.

[11] G. S. Agarwal. Phys. Rev. Lett. 1984. V. 53. P. 1732.

[12] M. Tavis, F. W. Cummings. Phys. Rev. 1968. V. 170. P. 379; W. R. Mallory. Phys. Rev. 1969. V. 188. P. 1976.

[13] N. Bogoliubov, R. K. Bullough, J. Timonen. J. Phys. A. 1996. V. 29. P. 6305.

[14] A. Rybin, G. Kastelewicz, J. Timonen, N. Bogoliubov. J. Phys. A. 1998. V. 31. P. 4705.

[15] L. Amico, K. Hikami. Integrable spin-boson interaction in the Tavis-Cummings model from a generic boundary twist. cond-mat/0309680.

[16] J. Dukelsky, G. G. Dussel, C. Esebbag, S. Pittel. Phys. Rev. Lett. 2004. V. 93. P. 050403.

[17] P. Kulish, E. K. Sklyanin. Quantum spectral transform method. Recent developments. In: Integrable Quantum Field Theories. Proc. of the Symp. (Tvaerminne, Finland, 23-27 March, 1981). Lect. Notes Phys. V. 151. Eds. J. Hietarinta, C. Montonen. Berlin: Springer, 1982. P. 61; A. G. Izergin, V. E. Korepin. Lett. Math. Phys. 1984. V. 8. P. 259; B. O. Tapacoв. ТМ $\Phi$. 1985. T. 63. C. 175 ; L. D. Faddeev. Int. J. Mod. Phys. A. 1995. V. 10. P. 1845.

[18] A. Kundu. Phys. Rev. Lett. 1999. V. 82. P. 3936.

[19] A. Kundu. J. Phys. A. 2004. V. 37. P. L281.

[20] L. Takhtajan. Introduction to algebraic Bethe ansatz. In: Exactly Solvable Problems in Condensed Matter and Relativistic Filed Theory. Proc. Winter School, Int. Colloq. (Panchgani, India, January 30 - February 12, 1985). Lect. Notes Phys. V. 242. Eds. B. S. Shastry, S. S. Jha, V. Singh. Berlin: Springer, 1985. P. 175.

[21] V. Pasquier, H. Saleur. Nucl. Phys. B. 1990. V. 330. P. 523

[22] E. К. Склянин. Функц. анализ и его прилож. 1982. Т. 16. № 4. С. 27; 1983. Т. 17. № 4. C. 34 .

[23] E. K. Sklyanin, T. Takebe. Phys. Lett. A. 1996. V. 219. P. 217.

[24] A. Kundu. Integrable multi atom matter-radiation models without rotating wave approximation. cond-mat/0411166.

[25] Л. А. Тахтаджян, Л. Д. Фаддеев. УМН. 1979. Т. 34. № 5. С. 13.

[26] T. Takebe. J. Phys. A. 1992. V. 25. P. 1071; 1995. V. 28. P. 6675.

[27] K. An, J. J. Childs, R. R. Dasari, M. S. Feld. Phys. Rev. Lett. 1994. V. 73. P. 3375; K. An. J. Phys. Soc. Japan. 2003. V. 72. P. 811. 